\title{
Long-Term Education, Employment and Family Outcomes in Biliary Atresia Patients, Post-Kasai
}

1. Faculty of Life Sciences and Medicine, King's College London, London, UK.

2. Paediatric Liver, GI, Nutrition and Mowatlabs, King's College Hospital, London, UK.

\section{Background}

Biliary atresia (BA) is an idiopathic neonatal cholangiopathy, requiring Kasai portoenterostomy during infancy. If unsuccessful, liver transplantation (LT) is required. Between 23-44\% survive with native liver (SNL) into adulthood, most with chronic liver disease (CLD). ${ }^{1}$ Unemployment rates for adults post LT is estimated up to $55 \% .{ }^{2}$ This study aimed to synthesise information on education, employment and family outcomes for patients with BA who had SNL up to 16 years and had not received a transplant.

\section{Methods}

- We reviewed electronic patient records (EPR) for patients diagnosed with BA between 1980-96, who SNL > 16 years, until last follow-up.

- Inpatient notes and outpatient clinic letters were scanned and searched for key phrases relevant to Family Status, Employment Status and Education Status.

- Information on schooling was achieved by searching for 'school' 'education' 'college' in inpatient notes; for employment, 'work', 'employ', 'job'; for family, 'husband', 'wife', 'partner', 'child', 'pregnancy', 'boy/girlfriend'. Liver clinic letters stored on our EPR system were read in full. International Standard Classification of Jobs (ISCO) were used.

\section{Education Status}

For those with data $(n=40) 100 \%$ attended school.

- 27 students for whom there was data stayed in full time education until the age of 18, studying A Levels or BTEC exams. For 2 students finishing age was unclear. 2 further students left school at the age of 16 . For the remainder there was no data.

- Sixty-five percent $(n=26)$ attended university, 35\% ( $n=14)$ did not.

BA SNL Patients and Education Status

Ever Attended Schoo

In Education Until 18

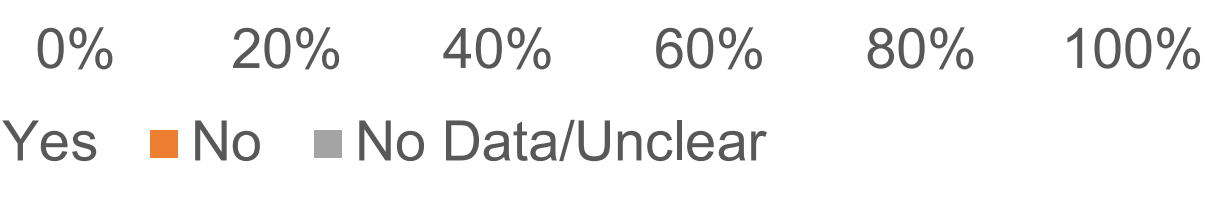

\section{Family Status}

- Overall $35 \%(n=25)$ of patients mentioned partners. For the remainder there was no information.

- Six women (of 35) had given birth at least once at last follow-up (17\%).

- The six women gave birth to a total of 12 children, at a median age of 25 years (range 21-35).

\section{Patient Characteristics}

- The cohort included $\mathbf{7 2}$ patients (49\% female), median age $\mathbf{2 5}$ years at last follow-up (range 16-36).

- Twenty-nine percent had portal hypertension (platelets<150x109/L) and jaundice (bilirubin $>20 u m o l / l)$.

\section{Employment Status}

- Ninety-six percent $(n=50)$ of those with data had held at least one job.

- Seven percent $(n=4)$ had never had a job.

- The patients were employed in a range of professions across various sectors.

- The most common type of employment was in service and sales. Overall $32 \%$ $(n=13)$ worked in service and sales, $29 \%(n=12)$ as professionals, $20 \%(n=8)$ as technicians, $7 \%(n=3)$ in elementary occupations, $5 \%(n=2)$ in craft and related trades, and $2 \%(n=1)$ each in plant and machine operators, skilled agricultural, and manager groups according to ISCO classifications.

- Sixty-eight percent $(n=15)$ worked full-time, $32 \%(n=7)$ worked part-time, with no data for the remainder.

\section{BA SNL Patients and Employment Status}

Ever Employed

$$
\begin{array}{ccccc}
0 \% & 20 \% & 40 \% & 60 \% & 80 \% \\
\text {-Yes } & - \text { No } & \text { - No Data/Unclear }
\end{array}
$$

BA SNL Patients and Professional Group

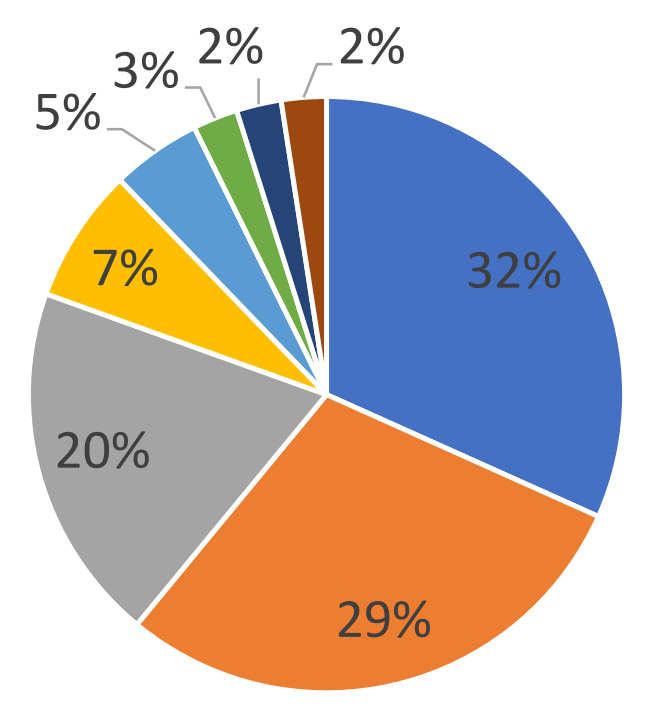

\section{Discussion}

Educational and employment outcomes for young adults with BA SNL in our centre are excellent with employment rates significantly better compared to adults with CLD and post LT. A multidisciplinary approach during adolescence can help young people to achieve their educational and professional potential.

- Regular screening with a questionnaire such as IMPARTS is recommended. IMPARTS is a questionnaire initiative trialed at King's College Hospital with subquestionnaires covering PHQ-9 (assessing depression), HADS (Hospital Anxiety and Depression Scale), AUDIT, WHODAS (quality of life), PHQ-15 (physical symptoms) and other areas assessing mental health and substance use. ${ }^{3}$

- Although research into these outcomes in this population thus far has been limited, the findings of this study were in agreement with others reporting high employment in patients with BA who SNL until adulthood, such as Wong et al. who found that 14 of 15 eligible adult patients were in work. ${ }^{4}$

\section{References}

= Ling, SC. Management of adult survivors of biliary atresia. Clinical Liver Disease, 2014. 4(4), 86-88.

= Aberg, F. From prolonging life to prolonging working life: Tackling unemployment among liver-transplant recipients. World Journal of Gastroenterology, 2016. 22(14), 3701-3711.

$3=$ The IMPARTS package for physical healthcare settings. Accessed $29^{\text {th }}$ September 2018. Available at: https://www.kcl.ac.uk/ioppn/depts/pm/research/imparts/Quick-links/IMPARTS-Package.pdf 